\title{
Expression and clinical significance of SALL4 and LGR5 in patients with lung cancer
}

\author{
AJAY KUMAR GAUTAM $^{1 *}$, CHANGMING WANG $^{1 *}$, JINRONG ZENG $^{1}$, JIYING WANG $^{1}$, JINGYAN LU $^{2}$,

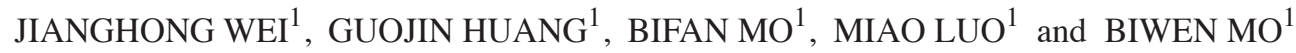 \\ Divisions of ${ }^{1}$ Respiratory Diseases and ${ }^{2}$ Pathology, Affiliated Hospital of Guilin Medical University, \\ Guilin, Guangxi 541001, P.R. China
}

Received November 4, 2014; Accepted September 4, 2015

DOI: $10.3892 / \mathrm{ol} .2015 .3772$

\begin{abstract}
Lung cancer is the most frequent cancer worldwide, in terms of incidence and mortality. Due to challenges in the diagnosis of the disease, the 5-year overall survival rate is only $\sim 16 \%$. Previous studies have suggested that malignant transformations originate from adult stem cells, and malignant lesions may therefore express stem-cell-associated markers. The purpose of the present study is to investigate the expression and clinical significance of the stem cell-associated markers Sal-like protein 4 (SALL4) and leucine-rich repeat-containing $\mathrm{G}$-protein coupled receptor 5 (LGR5) in lung cancer, and to provide novel diagnostic markers and targets for the treatment of lung cancer. The expression of the stem cell-associated markers SALL4 and LGR5 was analyzed by immunohistochemistry performed on 135 human lung cancer tissue specimens and 10 non-cancer lung tissue specimens. The clinical significance of the expression of these markers and correlation between their expression and clinical parameters was also assessed. SALL4 expression was highly upregulated in lung cancer tissues, but was not present in non-cancerous lung tissues, and the sensitivity and specificity of SALL4 reached $88 \%$ and $100 \%$, respectively. By contrast, LGR5 demonstrated $97 \%$ sensitivity, but the specificity was poor. Therefore,
\end{abstract}

Correspondence to: Dr Biwen Mo, Division of Respiratory Diseases, Affiliated Hospital of Guilin Medical University, 15 Lequn Road, Guilin, Guangxi 541001, P.R. China

E-mail: mobiwen@yahoo.com

*Contributed equally

Abbreviations: CSC, cancer stem cell; CRC, colorectal carcinoma; ESCC, esophageal squamous cell carcinoma; EAC, esophageal adenocarcinoma; BE, Barrett's esophagus; RT-PCR, reverse transcription polymerase chain reaction; GCT, germ cell tumor

Key words: stem-cell-associated marker, SALL4, LGR5, lung cancer, therapeutic target, diagnostic marker
SALL4 may be an extremely useful diagnostic marker for lung cancer, but LGR5 is not as useful.

\section{Introduction}

Lung cancer is the most common cancer worldwide, in terms of incidence and mortality, as it comprises $17 \%$ of total novel cancer cases and $23 \%$ of the total global cancer mortalities (1). In order to improve the survival rate, it is important to diagnose and surgically excise lung cancer at an early stage of disease (2). Therefore, it is necessary to identify biomarkers with the potential to facilitate tumor diagnosis, particularly in the early stages of the disease. The cancer stem cell (CSC) theory proposes that tumors contain a small subpopulation of CSCs, which are responsible for tumor growth, invasion and metastasis (2). CSCs and normal tissue stem cells share important characteristics, including self-renewal, multipotency and unlimited proliferation, and potentially possess overlapping molecular mechanisms $(3,4)$. Currently, a considerable number of stem cell-associated markers have been identified $(2,3)$. Numerous studies have revealed that Sal-like protein 4 (SALL4) and leucine-rich repeat-containing G-protein coupled receptor 5 (LGR5) are also involved in the tumorigenesis, development and metastasis of various tumors (5-7), and these proteins are expected to become potential diagnostic markers and therapeutic targets in cancer.

SALL4, a homolog of the Drosophila homeotic gene spalt, is a zinc-finger transcription factor that is required for the proliferation and maintenance of pluripotency through interaction with OCT3/4, sex determining region Y-box 2 and NANOG $(8,9)$. SALL4 is also highly expressed in embryonic stem cells (8-12). Notably, SALL4 is also overexpressed in various types of human hematopoietic malignancies, including acute myelocytic and lymphocytic leukemia $(5,13)$. In addition, SALL4 upregulates the expression of the oncogene Bmi-1 in human hematopoietic stem cells and leukemia cells (14). Numerous other studies have also revealed the oncogenic potential of SALL4 $(5,6,15)$, indicating that SALL4 may be used as a diagnostic marker in human malignancies. However, there have been no studies investigating the sensitivity and specificity of SALL4 expression in the diagnosis of non-germ cell tumors (GCTs). The present study analyzed the significance of SALL4 expression in lung cancer cells. 
LGR5, also termed G-protein coupled receptor 49 or G-protein coupled receptor 67 , is a protein that is encoded by the LGR5 gene in humans $(16,17)$. LGR5 is a member of the G-protein coupled receptor class A orphan receptor proteins. LGR5 is expressed across a diverse range of tissues, including the muscle, placenta, spinal cord and brain, and particularly acts as a biomarker of adult stem cells in certain tissues, such as adipose tissue and skeletal muscles (18). LGR5 was first identified by Hsu et al in Drosophila (18) and acts as a CSC marker for colorectal carcinoma (CRC). LGR5 is not only used as a biomarker, but is also likely to play an important role in maintaining the undifferentiated state of CSCs. Numerous studies have revealed that LGR5 acts as a CSC marker (19-21).

In the present study, the expression of SALL4 and LGR5 was examined in lung cancer and non-cancerous lung tissues using immunohistochemistry and the clinical significance and diagnostic value of these two tumor markers in lung cancer was evaluated.

\section{Materials and methods}

Lung cancer specimens. Tissue chips containing 135 specimens of lung cancer tissue, 5 specimens of normal lung tissue and 5 specimens of pneumonia-infected tissues were purchased from Guilin Fanpu Biotech (Guilin, Guangxi, China).

The age range of the patients that the tissues were obtained from was 31-78 years. In total, 102 patients were male and 33 patients were female. In addition, 91 patients were diagnosed with lymph node metastasis. The types of lung cancer that the present patients were diagnosed with are reported in Table I. Written informed consent was obtained from the patients, and the study was approved by the ethics committee of Guilin Medical University (Guilin, Guangxi, China).

Immunohistochemical staining. The paraffin-embedded specimens were sliced into $4 \mu \mathrm{m}$ sections. The slides were dried at $37^{\circ} \mathrm{C}$ overnight and the tissue sections were baked at $60^{\circ} \mathrm{C}$ for $2 \mathrm{~h}$. The slides were then deparaffinized with xylene and rehydrated using ethanol and immersed in 3\% hydrogen peroxide for 10 min to block endogenous peroxidase activity. An antigen retrieval process was accomplished by pressure cooking the slides at $120^{\circ} \mathrm{C}$ and pressure of $103 \mathrm{kPa}(15 \mathrm{psi})$ for $3 \mathrm{~min}$ in Tris/EDTA ( $\mathrm{pH}$ 8.0). The slides were then incubated with primary rabbit anti-human polyclonal antibodies against SALL4 (cat no. GTX109983; GeneTex, Inc., Irvine, CA, USA) and LGR5 (cat no. 21833-1-AP; ProteinTech, Chicago, IL, USA) for $1 \mathrm{~h}$ at room temperature in a moist chamber with saturated humidity. The dilutions used for the SALL4 and LGR5 antibodies were 1:100 and 1:600, respectively. The specimens were stained with 3,3'-diaminobenzidine subsequent to incubation with the secondary antibody (goat anti-rabbit IgG; 1:400 dilution; cat no. sc-2040; Santa Cruz Biotechnology, Inc., Dallas, TX, USA) for $30 \mathrm{~min}$. Finally, the sections were counterstained with hematoxylin, dehydrated and mounted. Confirmed biopsy specimens were used as a positive control. The slides treated with non-specific serum were used as a negative control.

Criteria for evaluation. The SALL4 protein was located in the cytoplasm and cell membrane. Positive cytoplasmic staining for SALL4 was indicated by yellow staining with cell shading.
Table I. Pathological types of lung cancer investigated in the present study.

Types of lung cancer

Cases, $\mathrm{n}$

Squamous cell carcinoma

64

Adenocarcinoma

Papillary adenocarcinoma

Adenosquamous carcinoma

Small cell carcinoma

Pulmonary arterial carcinoma

Metastatic carcinoma

Carcinoid tumor

Undifferential tumor

The LGR5 protein was located in the cytoplasm and nucleus. LGR 5 expression was indicated by brown staining. In total, 5 microscopic fields in each slide were randomly selected. Positive cells were counted under high magnification (magnification, x400) and the results were expressed according to the following criteria: 1 , Strong positive, $>75 \%$ tumor cells stained brown; 2 , mild positive, $25-75 \%$ of tumor cells stained medium brown; 3 , weak positive, $<25 \%$ of tumor cells stained pale yellow; and 4, absent, no expression.

All slides were independently reviewed by an experienced pathologist and researcher, without knowledge of the clinical data. Inconsistent cases were assessed again together.

Statistical analysis. Statistical analysis of results was performed using the SPSS 17.0 statistical package (SPSS, Inc., Chicago, IL, USA). The expression rate of stem cell-associated markers in lung cancer and the difference between the expression rates in the benign lung tissue specimens were analyzed using Fisher's exact test. The association between the expression of stem cell-associated markers in lung cancer and clinicopathological factors was analyzed using the $\chi^{2}$ test or Fisher's exact test, and the Mann-Whitney U test or Kruskal-Wallis H test. The correlation between two stem cell-associated markers was analyzed using Spearman's rank correlation coefficient. $\mathrm{P}<0.05$ was considered to indicate a statistically significant difference.

\section{Results}

SALL4 is expressed in lung cancer tissues. The expression of the SALL4 protein was observed mainly in the cytoplasm and cell membrane of lung cancer cells, but SALL4 was not expressed in normal or inflammatory lung tissues, or in normal squamous epithelium (Fig. 1). Mild to weak SALL4 expression was observed in small cell lung cancer tissues. Strong to mild expression was observed in lung adenocarcinoma tissues, and strong to weak expression was observed in squamous cell carcinoma tissues (Fig. 1). In the present study, SALL4 expression was observed in 119 out of 135 tissue samples and the rate of SALL4 expression was $88 \%$ (Table II).

LGR5 is expressed in non-cancerous cells, consisting of normal lung cells and benign lung lesions, and lung cancer 
Table II. The expression of SALL4 and LGR5 in each group.

\begin{tabular}{lccr}
\hline Markers & $\begin{array}{c}\text { Expression in normal and } \\
\text { non-cancerous tissues, } \mathrm{n}(\%)\end{array}$ & $\begin{array}{c}\text { Expression in lung } \\
\text { cancer tissues, } \mathrm{n}(\%)\end{array}$ & $\chi^{2}$ \\
\hline Total & $10(100)$ & $135(100)$ & 3.530 \\
SALL4 & $0(0)$ & $119(88)$ & 0.003 \\
LGR5 & $10(100)$ & $131(97)$ & 0.948 \\
\hline
\end{tabular}

SALL4, Sal-like protein 4; LGR5, leucine-rich repeat-containing G-protein coupled receptor 5.

Table III. Association between SALL4 expression and the clinical features of patients with lung cancer.

\begin{tabular}{|c|c|c|c|c|}
\hline Feature & Total & SALL4 expression, n (\%) & $\chi^{2}$ & P-value \\
\hline \multicolumn{5}{|l|}{ Age } \\
\hline$<60$ years & 80 & $67(83.75)$ & 2.386 & 0.122 \\
\hline$\geq 60$ years & 55 & $51(92.73)$ & & \\
\hline \multicolumn{5}{|l|}{ Gender } \\
\hline Male & 102 & $91(89.22)$ & 1.240 & 0.266 \\
\hline Female & 33 & $27(81.82)$ & & \\
\hline \multicolumn{5}{|l|}{ Histology } \\
\hline Adenocarcinoma & 35 & $32(91.43)$ & 2.703 & 0.609 \\
\hline Squamous cell carcinoma & 64 & $58(90.63)$ & & \\
\hline Adenosquamous carcinoma & 9 & $7(77.78)$ & & \\
\hline Papillary adenocarcinoma & 9 & $7(77.78)$ & & \\
\hline \multicolumn{5}{|l|}{ Lymph node metastasis } \\
\hline Yes & 41 & $36(81.80)$ & 0.004 & 0.95 \\
\hline No & 93 & $82(88.17)$ & & \\
\hline \multicolumn{5}{|l|}{ Stage } \\
\hline I-II & 48 & $45(93.75)$ & 0.003 & 0.959 \\
\hline III & 50 & $47(94.00)$ & & \\
\hline
\end{tabular}

SALL4, Sal-like protein 4.

cells. Medium expression of the LGR5 protein was identified in normal and inflammatory lung tissues, but LGR5 was not expressed in normal squamous epithelium, and was only weakly expressed in macrophages. Strong to mild LGR5 expression was observed in small cell lung cancer tissues. Mild to weak LGR5 expression was observed in lung adenocarcinoma and squamous cell lung carcinoma tissues (Fig. 2). In the present study, LGR5 expression in lung cancer cells was observed in 131 out of 135 tissue samples. The rate of LGR5 expression was 97\% (Table II).

Association between the present of lung cancer cell-associated markers and clinical features. In order to identify the association between these markers and the clinical features of patients with lung cancer, a $\chi^{2}$ test was performed. Since the numbers of analyzed cases of metastatic cancer, carcinoid tumor and differential carcinoma were too small, these conditions were not included when analyzing the association. The expression of SALL4 was not significantly associated with the patient gender or age, lymph node metastasis, pathological type of cancer or lung cancer stage $(\mathrm{P}>0.05)$. This association is reported in detail in Table III. The expression of LGR5 was significantly associated with patient gender $(\mathrm{P}<0.05)$, but was not significantly associated with the patient age, presence of lymph node metastasis, pathological type of cancer or lung cancer stage $(\mathrm{P}>0.05)$. This association is reported in detail in Table IV.

Correlation between SALL4 and LGR5. Spearman's rank correlation analysis revealed that the expression of SALL4 was not significantly correlated with the expression of LGR5 in lung carcinoma tissues [correlation coefficient $(\mathrm{R} / \mathrm{P}), 1 / 0.170 ; \mathrm{P}>0.05$; Table V].

Sensitivity and specificity of SALL4 and LGR5 expression in lung cancer cells. SALL4 was found to be expressed in $88 \%$ of lung cancer tissue specimens and LGR5 was expressed in $97 \%$ of lung cancer tissue specimens. High specificity of SALL4 was identified, but specificity of LGR5 was not observed (Table VI). 
Table IV. Correlation between LGR5 protein expression and the clinical features of patients with lung cancer.

\begin{tabular}{|c|c|c|c|c|}
\hline Feature & Total & LGR5 expression, n (\%) & $\chi^{2}$ & P-value \\
\hline \multicolumn{5}{|l|}{ Age } \\
\hline$<60$ years & 80 & $76(95.00)$ & 2.870 & 0.90 \\
\hline$\geq 60$ years & 55 & $55(100.00)$ & & \\
\hline \multicolumn{5}{|l|}{ Gender } \\
\hline Male & 102 & $101(99.02)$ & 5.704 & 0.017 \\
\hline Female & 33 & $30(90.91)$ & & \\
\hline \multicolumn{5}{|l|}{ Histology } \\
\hline Adenocarcinoma & 35 & $34(97.14)$ & 2.343 & 0.673 \\
\hline Squamous cell carcinoma & 64 & $62(96.88)$ & & \\
\hline Adenosquamous carcinoma & 9 & $9(100.00)$ & & \\
\hline Papillary adenocarcinoma & 9 & $8(88.89)$ & & \\
\hline \multicolumn{5}{|l|}{ Lymph node metastasis } \\
\hline Yes & 41 & $40(97.56)$ & 0.061 & 0.805 \\
\hline No & 93 & $90(96.77)$ & & \\
\hline
\end{tabular}

LGR5, leucine-rich repeat-containing G-protein coupled receptor 5.

Table V. Correlation between SALL4 and LGR5 expression.

\begin{tabular}{lccc}
\hline Protein & Correlation & $\begin{array}{c}\text { SALL4 } \\
\mathrm{R} / \mathrm{P}\end{array}$ & $\begin{array}{c}\text { LGR5 } \\
\mathrm{R} / \mathrm{P}\end{array}$ \\
\hline SALL4 & $\mathrm{R} / \mathrm{P}$ & 1.000 & $0.170^{\mathrm{a}}$ \\
LGR5 & $\mathrm{R} / \mathrm{P}$ & $0.170^{\mathrm{a}}$ & 1.000 \\
\hline
\end{tabular}

${ }^{\mathrm{a}} \mathrm{P}=0.005$. SALL4, Sal-like protein 4; LGR5, leucine-rich repeat-containing G-protein coupled receptor 5.

Table VI. Comparison of the sensitivity and specificity of SALL4 and LGR5 expression in lung cancer tissues.

\begin{tabular}{lcc}
\hline Protein & Sensitivity, \% & Specificity, $\%$ \\
\hline SALL4 & 88 & 100 \\
LGR5 & 97 & 0 \\
\hline
\end{tabular}

SALL4, Sal-like protein 4; LGR5, leucine-rich repeat-containing G-protein coupled receptor 5 .

\section{Discussion}

The present study investigated the expression and clinical significance of SALL4 and LGR5 expression in lung cancer by immunohistochemistry, and also explored the possible use of the SALL4 and LGR5 proteins as diagnostic markers for lung cancer. The present results demonstrated that SALL4 and LGR5 are each highly expressed in lung cancer. SALL4 was found to be expressed in $88 \%$ of lung cancer samples and LGR5 in 97\% of lung cancer samples. High specificity of SALL4 was also identified, but specificity of LGR5 was not observed, indicating that SALL4 may be used as an important diagnostic marker for lung cancer due to the specific and high expression in lung cancer cells.

Similar to numerous other studies $(3,5,20)$, the present study supports the hypothesis that cancer cells exhibit CSC markers, and certain CSC markers may demonstrate considerable clinical importance in lung cancer.

Expression analysis of SALL4 in non-cancerous and cancerous lung tissues revealed that SALL4 is overexpressed in $88 \%$ of lung cancer tissue samples (119 out of 135 cases). Similar studies have also reported the overexpression of SALL4 in various malignancies (21-23). In breast cancer, it has been demonstrated that the SALL4 mRNA level is elevated in $86.1 \%$ of tumor samples (21). In addition, the increase was observed even in the early stages of tumors (24). However, there was no significant correlation between the clinicopathological features of the patients and SALL4 mRNA expression in breast cancer (22). Analysis of SALL4 expression in normal and tumor colorectal tissues revealed that SALL4 is overexpressed in almost $90 \%$ of CRC samples, in which SALL4 expression was significantly associated with tumor cell metastasis to lymph nodes and the grade of tumor cell differentiation (23). It has been revealed that the expression of SALL4 in all types of testicular GCTs is associated with the degree of tumor differentiation, and it has been suggested that SALL4 is essential for the maintenance of the poorly differentiated status of testicular GCTs (15). In addition, the expression of SALL4 was detected in $>90 \%$ of the tumor cells of metastatic seminomas, dysgerminomas and embryonal carcinomas, indicating that SALL4 plays a role in the development of GCTs. Based on these results, SALL4 has been suggested as a novel sensitive and specific marker for metastatic GCTs and as a novel diagnostic marker for metastatic yolk sac tumors from the testis, ovary and extra gonadal sites (6). A previous study detected the expression of SALL4 at the mRNA level in cancerous and 

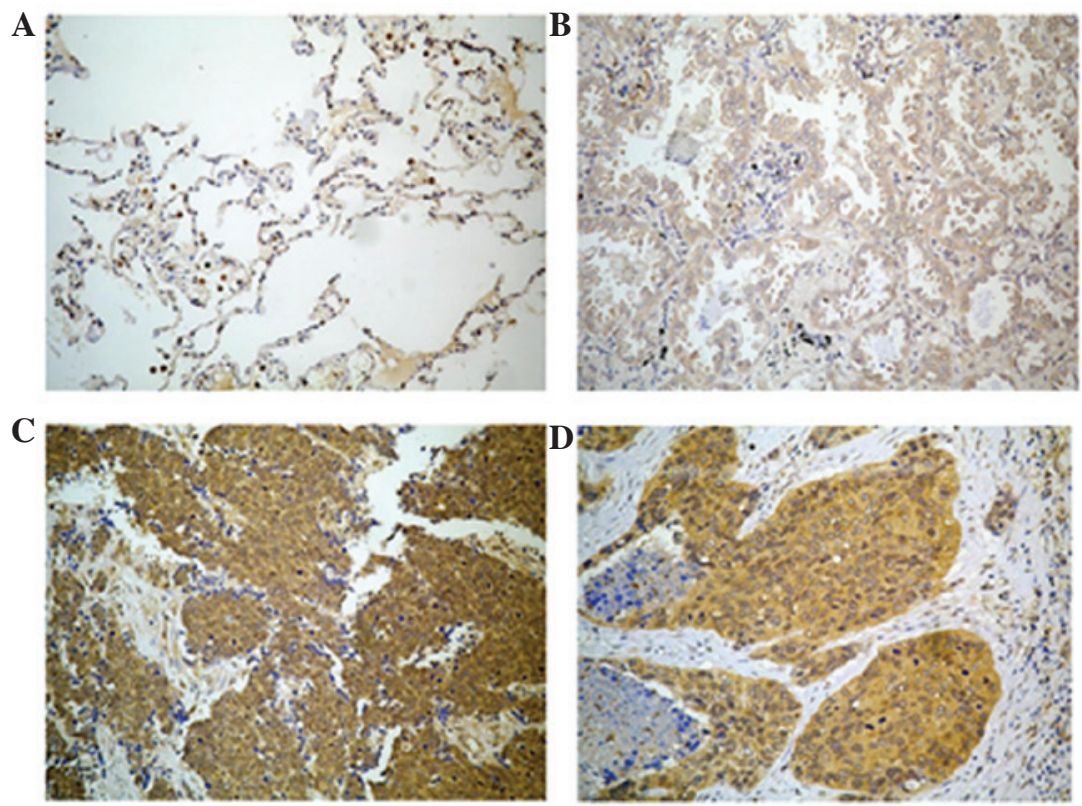

Figure 1. SALL4 expression in lung cancer tissues. (A) No expression of SALL4 in normal lung tissue. (B) Weak expression of SALL4 in adenocarcinoma tissue. (C) Strong expression of SALL4 in small cell carcinoma tissue. (D) Strong expression of SALL4 in squamous cell carcinoma tissue. Representative immunohistochemistry staining images are shown (magnification, x400). SALL4, Sal-like protein 4.
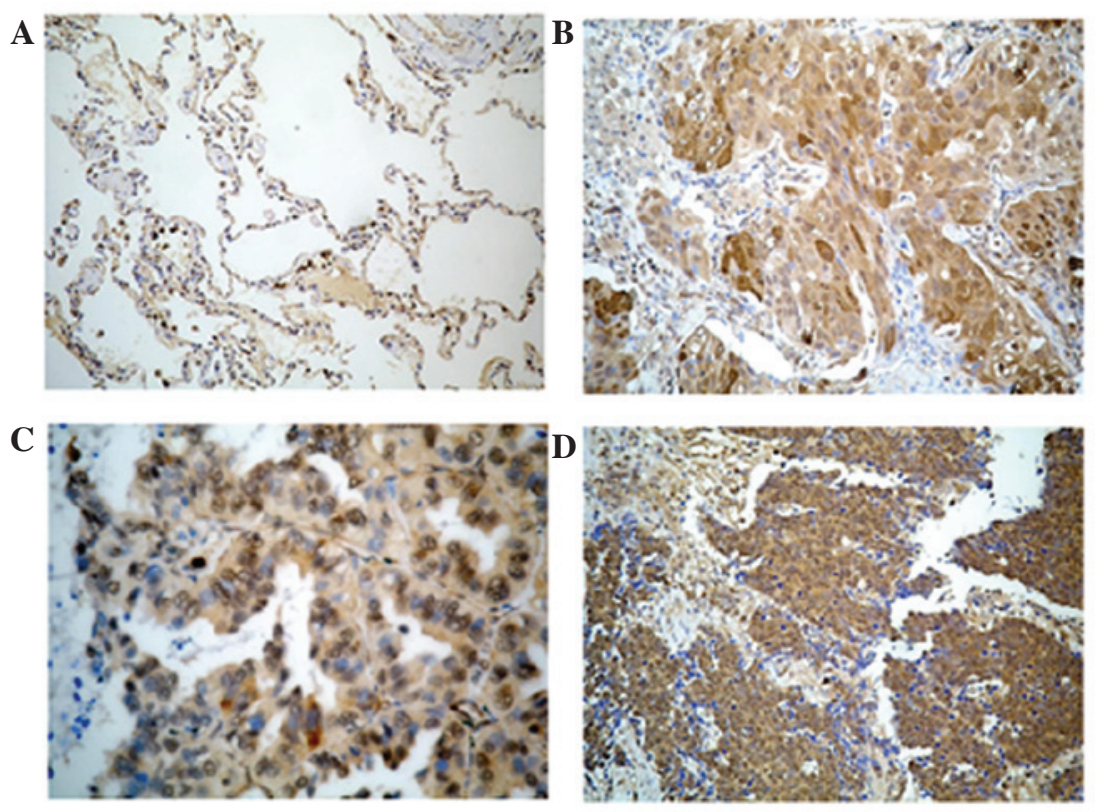

Figure 2. LGR5 is expressed in non-cancerous cells, consisting of normal lung cells and benign lung lesions, and lung cancer cells. (A) Mild expression of LGR5 in normal lung tissue. (B) Mild epression of LGR5 in squamous cell carcinoma inflammatory lung tissue. (C) Strong LGR5 expression in adenocarcinoma tissue. (D) Strong LGR5 expression in small cell carcinoma tissue. Representative immunohistochemistry staining images are shown (magnification, $\mathrm{x} 400$ ).

non-cancerous lung cancer tissues using reverse transcription-polymerase chain reaction (RT-PCR) (24). This study found that SALL4 mRNA expression was present in $80.9 \%$ of the cancerous tissues ( 38 of 47 ), and revealed that the sensitivity and specificity of SALL4 mRNA were 85.1 and $92.9 \%$, respectively (24).

In the present study, analysis of LGR5 protein expression in non-cancerous and cancerous lung tissues demonstrated that LGR5 is overexpressed in almost $97 \%$ of lung tissue samples. The current study found that LGR5 was significantly overexpressed in 131 out of 135 lung cancer tissue samples. However, the expression of LGR5 was also detected in non-cancerous tissue samples, consisting of 5 normal lung tissues, 4 tissue samples of lung infections and one tubercle nodule.

LGR5 acts as a CSC marker in CRC, and is likely to play an important role in maintaining the undifferentiated state of CSCs. In a previous study, LGR5 was found to be expressed in 35 out of 41 (85\%) esophageal adenocarcinoma (EAC) tissues with Barrett's esophagus (BE) and in 16 out of 
$19(81 \%)$ EAC tissues without BE. By contrast, LGR5 was not found to be expressed in esophageal squamous cell carcinoma tissues (25). In another study, RT-quantitative PCR analysis was performed on a panel of representative cancer cell lines derived from various organs, consisting of 11 colon cancer, 5 human hepatocellular carcinoma, 10 ovarian cancer and 11 lung cancer cell lines (26). LGR5 expression in each of the cell lines was normalized in the 37 cell lines on average. LGR5 mRNA was overexpressed in 5 out of 11 colon cancer cell lines, whereas LGR5 overexpression was rare in the cell lines derived from other cancers (27). Several studies have already focused on the effects of LGR5 expression in the context of tumor development and progression. LGR5 has been demonstrated to be involved in the pathogenesis of various human cancers, including hepatocellular carcinoma (26), basal cell carcinoma (28), endometrial cancer (29), colon cancer and ovarian cancer (7). Numerous studies have revealed that LGR5 is also expressed in adenoma and CRC cells $(18,26,27)$. These findings suggest that LGR5 may be a marker for intestinal stem cells, as well as for colorectal adenoma and carcinoma in humans.

All the aforementioned studies, including the present study, indicate the notable association between CSCs and cancer, in addition to the importance of various tumor markers in the diagnosis of various cancers. Overall, these findings and the present data indicate that the SALL4 expression may be a novel diagnostic marker for lung cancer. LGR5 expression is not an effective diagnostic marker for lung cancer due to its poor specificity for lung cancer. Additional investigation of the diagnostic potential of SALL4 in the early stages of lung cancer may have a profound clinical impact.

\section{Acknowledgements}

This study was supported by the Divisions of Pathology and Respiratory Disease of the Affiliated Hospital of Guilin Medical University. This study was also supported by the Key Research Project Grant of Guangxi Health Department (grant no. 2012003).

\section{References}

1. Jemal A, Bray F, Center MM, et al: Global cancer statistics. CA Cancer J Clin 61: 69-90, 2011.

2. Reya T, Morrison SJ, Clarke MF and Weissman IL: Stem cells, cancer and cancer stem cells. Nature 414: 105-111, 2001.

3. Visvader JE and Lindeman GJ: Cancer stem cells in solid tumours: Accumulating evidence and unresolved questions. Nat Rev Cancer 8: 755-768, 2008.

4. Hassan KA, Chen G, Kalemkerian GP, Wicha MS and Beer DG: An embryonic stem cell-like signature identifies poorly differentiated lung adenocarcinoma but not squamous cell carcinoma. Clin Cancer Res 15: 6386-6390, 2009.

5. Cui W, Kong NR, Ma Y, et al: Differential expression of the novel oncogene, SALL4, in lymphoma, plasma cell myeloma and acute lymphoblastic leukemia. Mod Pathol 19: 1585-1592, 2006.

6. Cao D, Humphrey PA and Allan RW: SALL4 is a novel sensitive and specific marker for metastatic germ cell tumors, with particular utility in detection of metastatic yolk sac tumors. Cancer 115: 2640-2651, 2009.

7. McClanahan T, Koseoglu S, Smith K, et al: Identification of overexpression of orphan $\mathrm{G}$ protein-coupled receptor GPR49 in human colon and ovarian primary tumors. Cancer Biol Ther 5 : 419-426, 2006.
8. Sakaki-Yumoto M, Kobayashi C, Sato A, et al: The murine homolog of SALL4, a causative gene in Okihiro syndrome, is essential for embryonic stem cell proliferation and cooperates with Sall1 in anorectal, heart, brain and kidney development. Development 133: 3005-3013, 2006.

9. Elling U, Klasen C, Eisenberger T, Anlag K and Treier M: Murine inner cell mass-derived lineages depend on Sall4 function. Proc Natl Acad Sci USA 103: 16319-16324, 2006.

10. Zhang J, Tam WL, Tong GQ, et al: Sall4 modulates embryonic stem cell pluripotency and early embryonic development by the transcriptional regulation of Pou5f1. Nat Cell Biol 8: 1114-1123, 2006.

11. Lim CY, Tam WL, Zhang J, et al: Sall4 regulates distinct transcription circuitries in different blastocyst-derived stem cell lineages. Cell Stem Cell 3: 543-554, 2008.

12. Yang J, Gao C, Chai L and Ma Y: A novel SALL4/OCT4 transcriptional feedback network for pluripotency of embryonic stem cells. PLoS One 5: e10766, 2010.

13. Yang J, Chai L, Gao C, et al: SALL4 is a key regulator of survival and apoptosis in human leukemic cells. Blood 112: 805-813, 2008.

14. Yang J, Chai L, Liu F, et al: Bmi-1 is a target gene for SALL4 in hematopoietic and leukemic cells. Proc Natl Acad Sci USA 104: 10494-10499, 2007.

15. Cao D, Li J, Guo CC, et al: SALL4 is a novel diagnostic marker for testicular germ cell tumors. Am J Surg Pathol 33: 1065-1077, 2009.

16. McDonald T, Wang R, Bailey W, et al: Identification and cloning of an orphan $\mathrm{G}$ protein-coupled receptor of the glycoprotein hormone receptor subfamily. Biochem Biophys Res Commun 247: 266-270, 1998.

17. McClanahan T, Koseoglu S, Smith K, et al: Identification of overexpression of orphan $\mathrm{G}$ protein-coupled receptor GPR49 in human colon and ovarian primary tumors. Cancer Biol Ther 5: 419-426, 2006.

18. Hsu SY, Liang SG and Hsueh AJ: Characterization of two LGR genes homologous to gonadotropin and thyrotropin receptors with extracellular leucine-rich repeats and a G-protein-coupled, seven-transmembrane region. Mol Endocrinol 12: 1830-1845, 1998.

19. Vermeulen L, Todaro M, de Sousa Mello F, et al: Single-cell cloning of colon cancer stem cells reveals a multi-lineage differentiation capacity. Proc Natl Acad Sci USA 105: 13427-13432, 2008.

20. Barker N, Ridgway RA, van Es JH, et al: Crypt stem cells as the cells of-origin of intestinal cancer. Nature 457: 608-611, 2009.

21. Takashima S, Kadowaki M, Aoyama K, et al: The Wnt agonist R-spondin1 regulates systemic graft-versus-host disease by protecting intestinal stem cells. J Exp Med 208: 285-294, 2011.

22. Kobayashi D, Kuribayshi K, Tanaka M and Watanabe N: SALL4 is essential for cancer cell proliferation and is overexpressed at early clinical stages in breast cancer. Int J Oncol 38: 933-939, 2011

23. Forghanifard MM, Moghbeli M, Raeisossadati R, et al: Role of SALL4 in the progression and metastasis of colorectal cancer. J Biomed Sci 20: 6, 2013.

24. Kobayashi D, Kuribayashi K, Tanaka M and Watanabe N: Overexpression of SALL4 in lung cancer and its importance in cell proliferation. Oncol Rep 26: 965-970, 2011.

25. von Rahden BH, Kircher S, Lazariotou M, et al: LgR5 expression and cancer stem cell hypothesis: Clue to define the true origin of esophageal adenocarcinomas with and without Barrett's Esophagus? J Exp Clin Cancer Res 30: 23, 2011.

26. Yamamoto Y, Sakamoto M, Fujii G, et al: Overexpression of orphan G-protein-coupled receptor, Gpr49, in human hepatocellular carcinomas with beta-catenin mutations. Hepatology 37: 528-533, 2003.

27. Uchida H, Yamazaki K, Fukuma M, et al: Overexpression of leucine-rich repeat-containing Gprotein-coupled receptor 5 in colorectal cancer. Cancer Sci 101: 1731-1737, 2010.

28. Tanese K, Fukuma M, Yamada T, et al: G-protein-coupled receptor GPR49 is up-regulated in basal cell carcinoma and promotes cell proliferation and tumor formation. Am J Pathol 173: 835-843, 2008

29. Sun X, Jackson L, Dey SK and Daikoku T: In Pursuit of leucine-rich repeat-containing $G$ protein-coupled receptor-5 regulation and function in the uterus. Endocrinology 150: 5065-5073, 2009. 